$1-1-2016$

\title{
Racemose neurocysticercosis.
}

\author{
Kristin Krupa \\ Sidney Kimmel Medical College, Thomas Jefferson University \\ Kelly Krupa, BS \\ Sidney Kimmel Medical College, Thomas Jefferson University \\ Mary L Pisculli \\ Department of Neurological Surgery, Thomas Jefferson University \\ Deena M Athas \\ Division of Infectious Diseases and Environmental Medicine, Thomas Jefferson University \\ Christopher J Farrell, MD \\ Department of Neurological Surgery, Thomas Jefferson University
}

Follow this and additional works at: https://jdc.jefferson.edu/neurosurgeryfp

Part of the Other Medical Specialties Commons

Let us know how access to this document benefits you

\section{Recommended Citation}

Krupa, Kristin; Krupa, BS, Kelly; Pisculli, Mary L; Athas, Deena M; and Farrell, MD, Christopher J,

"Racemose neurocysticercosis." (2016). Department of Neurosurgery Faculty Papers. Paper 79.

https://jdc.jefferson.edu/neurosurgeryfp/79

This Article is brought to you for free and open access by the Jefferson Digital Commons. The Jefferson Digital Commons is a service of Thomas Jefferson University's Center for Teaching and Learning (CTL). The Commons is a showcase for Jefferson books and journals, peer-reviewed scholarly publications, unique historical collections from the University archives, and teaching tools. The Jefferson Digital Commons allows researchers and interested readers anywhere in the world to learn about and keep up to date with Jefferson scholarship. This article has been accepted for inclusion in Department of Neurosurgery Faculty Papers by an authorized administrator of the Jefferson Digital Commons. For more information, please contact: JeffersonDigitalCommons@jefferson.edu. 


\title{
Case Report
}

\section{Racemose neurocysticercosis}

\author{
Kristin Krupa, Kelly Krupa, Mary L. Pisculli ${ }^{1}$, Deena M. Athas², Christopher J. Farrell ${ }^{1}$ \\ Sidney Kimmel Medical College, Thomas Jefferson University, ${ }^{1}$ Department of Neurological Surgery, Thomas Jefferson University, ${ }^{2}$ Division of Infectious Diseases \\ and Environmental Medicine, Thomas Jefferson University, Philadelphia, PA, USA \\ E-mail: Kristin Krupa - kristin.krupa@jefferson.edu; Kelly Krupa - kelly.krupa@jefferson.edu; Mary L.Pisculli - christopher.farrell@jefferson.edu; \\ Deena M. Athas - deena.athas@jefferson.edu; *Christopher J. Farrell - Christopher.Farrell@jefferson.edu \\ *Corresponding author
}

Received:09 May $15 \quad$ Accepted:01 October $15 \quad$ Published: 05 February 16

\begin{abstract}
Background: Neurocysticercosis (NCC) is an invasive parasitic infection of the central nervous system caused by the larval stage of the tapeworm Taenia solium. The clinical manifestations of NCC depend on the parasitic load and location of infection, as well as the developmental stage of the cysticerci and host immune response, with symptoms ranging from subclinical headaches to seizures, cerebrovascular events, and life-threatening hydrocephalus. Racemose NCC represents a particularly severe variant of extraparenchymal NCC characterized by the presence of multiple confluent cysts within the subarachnoid space and is associated with increased morbidity and mortality, as well as a decreased response to treatment. Albendazole is the preferred drug for the treatment of racemose NCC due to its superior cerebrospinal fluid penetration compared to praziquantel and the ability to be used concomitantly with steroids.

Case Description: In this report, we describe a 39-year-old man recently emigrated from Mexico with racemose NCC and hydrocephalus successfully treated with prolonged albendazole treatment, high-dose dexamethasone, and ventriculoperitoneal shunt placement for the relief of obstructive hydrocephalus.

Conclusions: Treatment of racemose NCC represents a significant clinical challenge requiring multimodal intervention to minimize infectious- and treatment-related morbidity. We review the clinical, diagnostic, and therapeutic features relevant to the management of this aggressive form of NCC.
\end{abstract}

Key Words: Neurocysticercosis, racemose, Taenia solium, treatment

\begin{tabular}{|l|}
\hline Access this article online \\
\hline Website: \\
www.surgicalneurologyint.com \\
\hline DOI: \\
10.4103/2152-7806.175881 \\
\hline Quick Response Code: \\
\hline
\end{tabular}

\section{INTRODUCTION}

Neurocysticercosis (NCC) is a common parasitic infection of the central nervous system (CNS) caused by the tapeworm Taenia solium. Infection is typically characterized by the presence of intraparenchymal cysts within the cerebral hemispheres at the gray-white junction and NCC is the most frequent cause of acquired epilepsy in the developing countries. ${ }^{[17]}$ Parasitic infestation is endemic to South and Central America, Southeast Asia, China, and Sub-Saharan Africa ${ }^{[6]}$ and is of increasing importance in the United States due to high volumes of immigration from these endemic areas. ${ }^{[6,14,24]}$ Extraparenchymal NCC is less common than the intraparenchymal form and is defined as NCC involving the subarachnoid, meningeal, and intraventricular space. Extraparenchymal NCC presents

This is an open access article distributed under the terms of the Creative Commons Attribution-NonCommercial-ShareAlike 3.0 License, which allows others to remix, tweak, and build upon the work non-commercially, as long as the author is credited and the new creations are licensed under the identical terms.

For reprints contact: reprints@medknow.com

How to cite this article: Krupa K, Krupa K, Pisculli ML,Athas DM, Farrell CJ. Racemose neurocysticercosis. Surg Neurol Int 2016;7:12.

http://surgicalneurologyint.com/Racemose-neurocysticercosis/ 
unique diagnostic and therapeutic challenges compared to the intraparenchymal form. The racemose variant of extraparenchymal NCC represents a particularly aggressive infection and is associated with relatively increased morbidity and mortality and decreased responsiveness to medical treatment. The variant is characterized by the presence of multilobulated confluent cysts within the basal subarachnoid space that appear "grape-like" both radiographically and pathologically. ${ }^{[6]}$ In this report, we describe the successful management of a patient with extensive racemose NCC and hydrocephalus treated with cerebrospinal fluid (CSF) diversion and prolonged administration of albendazole and corticosteroids with complete radiographic and clinical resolution of infection.

\section{CASE REPORT}

A 39-year-old male presented to the emergency room with a complaint of worsening headaches and visual decline. The patient emigrated from Mexico 13 months prior to presentation and reported no other significant medical history. He described diffuse headaches that had progressed over a l-month period with new-onset bilateral visual impairment and nausea for the last week. His neurologic exam was nonfocal, however, the ophthalmologic evaluation revealed bilateral papilledema and diminished visual acuity. He was afebrile on presentation and without meningeal irritation signs. Initial laboratory findings indicated a normal white blood cell count of $7400 / \mathrm{mm}^{3}$ with $73 \%$ polymorphonuclear leukocytes, $21 \%$ lymphocytes, and $1 \%$ eosinophils. A head computed tomography (CT) scan demonstrated hydrocephalus with transependymal flow and a multilobulated hypodense mass in the basal cisterns. Subsequent magnetic resonance imaging (MRI) was performed which demonstrated the presence of too numerous to count nonenhancing, T2-hyperintense cystic lesions in the lateral ventricles and subarachnoid spaces including the bilateral sylvian fissures and cerebellopontine angles, as well as the suprasellar, interpeduncular, and prepontine cisterns with the largest cyst measuring $21 \mathrm{~mm}$ in diameter and exerting mild mass effect upon the brainstem [Figure 1]. Minor sylvian fissure enhancement was noted bilaterally, however, magnetic resonance angiography (MRA) showed no large vessel stenosis or occlusion. A screening MRI of the spine demonstrated a small subarachnoid cystic lesion at the level of the conus and multiple septations at the S1-S2 level. A lumbar puncture was performed, and an elevated opening pressure was documented. CSF analysis revealed a mild lymphocytic pleocytosis with 60 white blood cells $/ \mathrm{mm}^{3}$ (96\% lymphocytes, $4 \%$ eosinophils). The initial CSF protein level was $106 \mathrm{mg} / \mathrm{dL}$ with a glucose level of $54 \mathrm{mg} / \mathrm{dL}$. CSF cysticercus antibody ELISA testing was positive, and a diagnosis of racemose NCC was

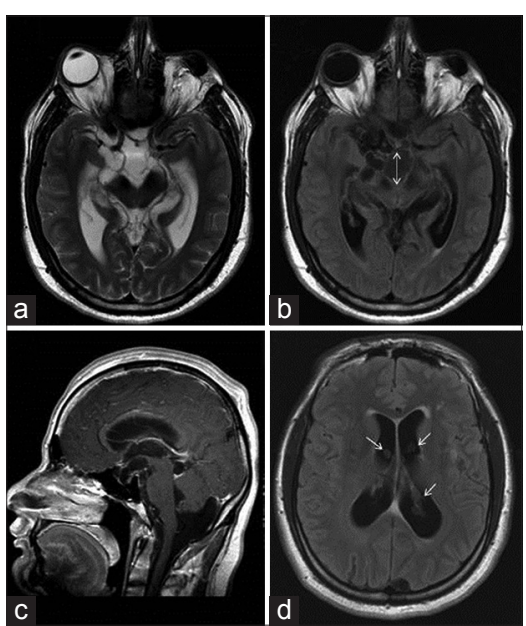

Figure I: (a) Axial T2-weighted magnetic resonance imaging demonstrating multiple confluent cystic lesions in the basal cisterns and sylvian fissures. (b) Fluid-attenuated inversion recovery magnetic resonance imaging showing multiple cystic lesions with the largest cyst measuring $21 \mathrm{~mm}$ in maximal diameter (dashed arrow) and mild mass effect on the brainstem. (c) Sagittal TI-weighted magnetic resonance imaging postgadolinium administration showing hydrocephalus and cystic lesions in suprasellar, prepontine, and foramen magnum cisterns. (d) Fluid-attenuated inversion recovery magnetic resonance imaging demonstrating multiple cystic lesions within the lateral ventricles bilaterally (arrows) with enlarged ventricles and transependymal flow

made based on the immunoassay results, neuroimaging findings, and a history of recent emigration from Mexico.

After extensive discussion between the neurosurgical and infectious diseases teams, we elected to proceed with placement of a ventriculoperitoneal shunt to ameliorate his elevated intracranial pressure and communicating hydrocephalus. High-dose dexamethasone (4 mg QID) was initiated followed by administration of prolonged albendazole $(10 \mathrm{mg} / \mathrm{kg} /$ day $)$ treatment. The patient experienced immediate relief of his visual deficit following the CSF diversion treatment. He maintained a mild headache disorder that responded well to steroids, and he was able to be slowly weaned off of this medication over a 12 month period. MRI scan performed at that time revealed complete radiographic resolution of his subarachnoid, spinal, and intraventricular cystic lesions with very mild persistent meningeal enhancement [Figure 2]. A serum cysticercus antigen ELISA test was nonreactive, and albendazole was discontinued.

\section{DISCUSSION}

Cysticercosis is the most common helminthic infection of the CNS with infection occurring primarily through the ingestion of eggs of the parasitic tapeworm T. solium. ${ }^{[13]}$ The eggs hatch within the digestive system and the released oncospheres penetrate the intestinal mucosa to enter the circulation. The oncospheres are then passively transported to various tissues including the brain, 

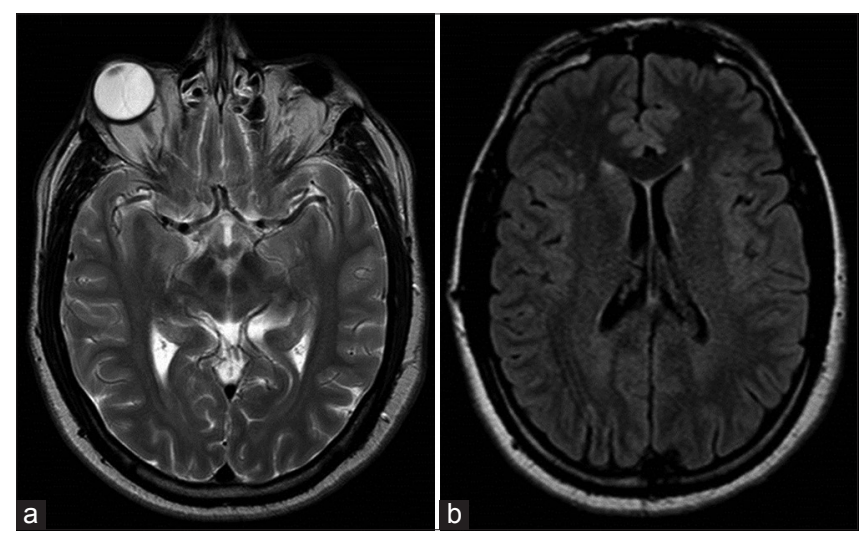

Figure 2: (a) Axial T2-weighted magnetic resonance imaging showing cyst resolution within the basal cisterns and sylvian fissures. (b) Fluid-attenuated inversion recovery magnetic resonance imaging showing resolution of the multiple cystic lesions within the lateral ventricles and hydrocephalus

muscle, and eyes where the parasites form larval cysts, or cysticerci. Extraparenchymal NCC occurs as the cysticerci reach the ventricles through the choroid plexus where they may pass freely or become attached to the ependyma. ${ }^{[16]}$ Intraventricular infection appears to be more frequent than previously believed with recent series documenting the presence of parasitic cysts in up to $30 \%$ of patients with NCC. ${ }^{[9]}$ Displacement of the cysts from the ventricles via the CSF may lead to infection within the basal cisterns, sylvian fissures, and cortical and spinal subarachnoid spaces. Excessive basal subarachnoid infection involving numerous parasitic membranes and enlarged vesicular cysts accompanied by a relative lack of scolices is termed racemose NCC, although no definitive diagnostic criteria for this rare but severe variant have been described. ${ }^{[6]}$

The diagnosis of racemose NCC is typically based on neuroimaging, serum and CSF laboratory evaluation, and clinical, epidemiologic data including immigration from or frequent travel to disease-endemic areas. Contrast-enhanced MRI is the imaging modality of choice for the detection of extraparenchymal NCC as CT has limited sensitivity for identification of intraventricular and small subarachnoid cysts. ${ }^{[16]}$ Lesions typically appear as hyperintense on T2-weighted sequences and provide a clear delineation of the cyst wall and the presence of obstructive or communicating hydrocephalus. Contrast evaluation is important to determine the extent of the host inflammatory response including ependymitis and basal arachnoiditis, as well as the evolutionary stage of the parasite. While the clinical and neuroimaging findings are of paramount importance, serologic testing may provide additional data, especially in patients without an appropriate epidemiologic history. Peripheral eosinophilia is a common but nonspecific finding with NCC. ${ }^{[8]}$ Similarly, the presence of CSF eosinophils is frequently indicative of NCC and is a helpful in differentiating NCC from other forms of infectious chronic meningitis, although this finding has been reported to occur in only $15 \%$ of patients. ${ }^{[9]}$ The most common CSF abnormalities observed with NCC are a mild-moderate lymphocytic pleocytosis and increase in protein with the levels varying according to parasitic burden and location of the infection. Positive serum and CSF immunoassays for detection of anticysticercal antibodies are highly suggestive of NCC infection in the appropriate clinical setting, although false-negative results are a well-recognized limitation. ${ }^{[8]}$ The sensitivity of these immunoassays has not been established for the racemose variant but would be expected to be increased given the robust inflammatory reaction typically elicited with subarachnoid infection.

The intraventricular and cisternal forms of NCC typically manifest in a more clinically aggressive manner than parenchymal infection and frequently cause intracranial hypertension due to CSF outflow obstruction or inflammatory basal arachnoiditis. ${ }^{[21]}$ Cysts within the ventricular cavities may be free floating and cause obstruction at the foramina of Monro, sylvian aqueduct, or fourth ventricle and may present with rapid clinical deterioration secondary to acute hydrocephalus. These cysts may also become attached to the ependymal wall of the ventricle and result in ependymitis following cyst degeneration that may lead to intraventricular loculation and make CSF diversion more problematic. Similarly, cyst degeneration within the subarachnoid space may elicit an accompanying host inflammatory response with basal arachnoiditis, vasculitis, elevated intracranial pressure, and subacute to chronic hydrocephalus requiring CSF diversion. In 2002, DeGiorgio et al. demonstrated that patients with heavy cyst burdens and hydrocephalus carried the highest risk of mortality with NCC, primarily because of elevated intracranial pressure and shunt-related problems. ${ }^{[7]}$ Similarly, Colli et al. reported a $30.8 \%$ mortality in patients who required placement of a ventriculoperitoneal shunt and a $>80 \%$ rate of shunt failure secondary to shunt obstruction due to proteinaceous debris. ${ }^{[4]}$ In addition to hydrocephalus, the active inflammatory process may lead to vasculitis and cerebrovascular complications. Angiography or MRA may reveal segmental narrowing or occlusion of large vessels within the basal cisterns and sylvian fissures, with the majority of infarcts occurring in the lenticulostriate artery distributions..$^{[3]}$ Basal meningitis may also cause cranial nerve dysfunction due to fibrous entrapment. ${ }^{[16]}$ Callacondo et al. recently described that extension of infection to the spinal subarachnoid space is very common in racemose NCC, with spinal involvement documented in 17 of 28 patients in their prospective study leading the authors to recommend that screening MRI of the spine be performed in basal subarachnoid disease to document spinal involvement, prevent complications, and monitor for recurrent infection. ${ }^{[2]}$ 
No consensus exists regarding the optimal treatment of racemose NCC including the duration of anthelmintic treatment and the role of surgical intervention. In patients presenting with hydrocephalus, the priority is relief of raised intracranial pressure. ${ }^{[14]}$ Obstructive hydrocephalus due to intraventricular cysts is best managed endoscopically via cyst removal or drainage and often obviates the need for permanent shunt placement. ${ }^{[8,19]}$ Intraventricular cysts may be freely mobile and imaging immediately prior to neuroendoscopic intervention is recommended to confirm the location of the cyst. A relative contraindication for endoscopic removal of intraventricular cysts is the presence of significant ependymal enhancement as this typically indicates dense adhesion between the parasitic cyst and the ependymal wall with attempted cyst removal associated with neurologic injury and intraventricular hemorrhage. ${ }^{[10,20]}$ A role for endoscopic or microsurgical removal of subarachnoid cysts within the basal cisterns and sylvian fissures is less established due to frequently high cyst burden and widespread subarachnoid distribution and inflammatory response. Giant $(>5 \mathrm{~cm})$ subarachnoid cysts with associated mass effect and intracranial hypertension are most often managed surgically, although Proaño et al. demonstrated complete resolution of giant cysts in 19 of 33 patients treated medically with prolonged multicourse anthelmintic and corticosteroid therapy. ${ }^{[18]}$ Couldwell et al. suggested that surgical therapy be reserved for cisternal forms of NCC if initial medical management fails or experiences neurologic decline as conventional microsurgical approaches have been associated with significant morbidity, likely due to the presence of arachnoiditis with adherence of the cyst walls to cranial nerves and arteries. ${ }^{[5]}$ More recently, several authors have described minimally invasive keyhole or endoscopic approaches to the basal cisterns to achieve cyst drainage, although the utility of these approaches for extensive subarachnoid disease has yet to be established. ${ }^{[1]}$ Fortunately, spillage of cyst contents with these approaches has not proven to exacerbate the inflammatory response in the postoperative period, although careful attention to this concern and copious intraoperative irrigation are recommended. ${ }^{[11,12]}$

In our patient with hydrocephalus and relatively rapid progression of visual loss, given the bilateral sylvian fissure involvement and absence of any dominant cystic lesion or focal neurologic deficits, we elected to proceed with placement of a ventriculoperitoneal shunt prior to initiating therapy directed against the invasive infection. Delayed exacerbation of intracranial hypertension frequently occurs several days following administration of cysticidal agents as a result of cyst degeneration and subsequent host inflammatory response with the release of proinflammatory cytokines. ${ }^{[6,23]}$ For this reason, steroids should be administered concurrently with anthelmintic therapy to reduce the inflammatory response. Subarachnoid cysts are considered less responsive to pharmacologic therapy compared to intraparenchymal lesions, with albendazole the preferred drug for treatment of racemose NCC due to its superior CSF penetration compared to praziquantel. ${ }^{[8]}$ Additionally, the CNS distribution of albendazole is less affected by steroid administration. ${ }^{[22]}$ The dose and duration of treatment must be individually tailored to each patient depending on their parasitic burden and clinical and radiographic response to treatment with the treatment course frequently longer than that prescribed for the parenchymal disease. Aggressive medical treatment has been shown to decrease the incidence of shunt malfunction, which has been directly linked to poorer clinical outcome. ${ }^{[15]}$ For patients with giant cysts, Proaño et al. achieved excellent results with a 4 weeks course of albendazole, although more than half of their patients required more than one treatment course to achieve cyst resolution. 4 of the 33 patients in their study required 3 or more months of therapy and 10 patients also received praziquantel following a failure of the cysts to respond to albendazole. ${ }^{[18]}$

\section{CONCLUSION}

In our patient, we reported the successful clinical resolution of racemose NCC following CSF diversion and prolonged anthelmintic therapy and corticosteroid administration to prevent the development of chronic subarachnoid inflammation. No shunt complications or revisions occurred, and a complete radiographic response was observed. Racemose NCC represents a, fortunately, rare but aggressive form of extraparenchymal NCC often resulting in basilar arachnoiditis, vasculitis, and cranial neuropathy. Proper recognition of this infection and appropriate management with careful consideration of the deleterious effects of the attendant host inflammatory response to cyst degeneration is necessary to reduce patient morbidity and mortality. Racemose NCC accompanied by intracranial hypertension and hydrocephalus is associated with poorer clinical outcomes, often related to ventriculoperitoneal shunt infection and malfunction. Vigilant clinical and radiographic monitoring of response to treatment is imperative to reduce neurologic sequelae and infection relapse as prolonged, or multicourse medical therapy is frequently necessary.

\section{Financial support and sponsorship Nil.}

Conflicts of interest

There are no conflicts of interest. 


\section{REFERENCES}

I. Angeles $\mathrm{CF}$,Vollmer D, Mohanty A. Transventricular neuroendoscopic excision of giant racemose subarachnoid cysticercosis. Childs Nerv Syst 2009;25:503-8.

2. Callacondo D, Garcia HH, Gonzales I, Escalante D, Nash TE; Cysticercosis Working Group in Peru. High frequency of spinal involvement in patients with basal subarachnoid neurocysticercosis. Neurology 20 I2;78: 1394-400.

3. Cantú C, Barinagarrementeria F. Cerebrovascular complications of neurocysticercosis. Clinical and neuroimaging spectrum. Arch Neurol 1996:53:233-9.

4. Colli BO, Carlotti CG Jr, Assirati JA Jr, Machado HR, Valença M,Amato MC. Surgical treatment of cerebral cysticercosis: Long-term results and prognostic factors. Neurosurg Focus 2002;12:e3.

5. Couldwell WT, Zee CS, Apuzzo ML. Definition of the role of contemporary surgical management in cisternal and parenchymatous cysticercosis cerebri. Neurosurgery 1991;28:231-7.

6. Das RR, Tekulve KJ, Agarwal A, Tormoehlen LM. Racemose neurocysticercosis. Semin Neurol 2012;32:550-5.

7. DeGiorgio CM, Houston I, Oviedo S, Sorvillo F. Deaths associated with cysticercosis. Report of three cases and review of the literature. Neurosurg Focus 2002;12:e2.

8. Del Brutto OH. Neurocysticercosis. Continuum (Minneap Minn) 2012;18:1392-416.

9. Figueroa JJ, Davis LE, Magalhaes A. Extraparenchymal neurocysticercosis in Albuquerque, New Mexico. J Neuroimaging 201 I;21:38-43.

10. Goel RK, Ahmad FU, Vellimana AK, Suri A, Chandra PS, Kumar R, et al. Endoscopic management of intraventricular neurocysticercosis. J Clin Neurosci 2008;15:1096-101.

II. Hawk MW, Shahlaie K, Kim KD, Theis JH. Neurocysticercosis: A review. Surg Neurol 2005;63:123-32.
12. Husain M, Jha DK, Rastogi M, Husain N, Gupta RK. Neuro-endoscopic management of intraventricular neurocysticercosis (NCC). Acta Neurochir (Wien) 2007;149:341-6.

13. Kelesidis T,Tsiodras S. Extraparenchymal neurocysticercosis in the United States. Am J Med Sci 20I2;344:79-82.

14. Kelesidis T,Tsiodras S. Extraparenchymal neurocysticercosis in the United States: A case report.J Med Case Rep 201 I;5:359.

15. Kelley R, Duong DH, Locke GE. Characteristics of ventricular shunt malfunctions among patients with neurocysticercosis. Neurosurgery 2002;50:757-6I.

16. Matushita H, Pinto FC, Cardeal DD, Teixeira MJ. Hydrocephalus in neurocysticercosis. Childs Nerv Syst 201 1;27:1709-2I.

17. Pal DK, Carpio A, Sander JW. Neurocysticercosis and epilepsy in developing countries.J Neurol Neurosurg Psychiatry 2000;68: 137-43.

18. Proaño JV, Madrazo I, Avelar F, López-Félix B, Díaz G, Grijalva I. Medical treatment for neurocysticercosis characterized by giant subarachnoid cysts. N Engl J Med 200 I;345:879-85.

19. Rangel-Castilla L, Serpa JA, Gopinath SP, Graviss EA, Diaz-Marchan P, White AC Jr. Contemporary neurosurgical approaches to neurocysticercosis. Am J Trop Med Hyg 2009;80:373-8.

20. Sinha S, Sharma BS. Intraventricular neurocysticercosis: A review of current status and management issues. Br J Neurosurg 2012;26:305-9.

21. Sinha S, Sharma BS. Neurocysticercosis: A review of current status and management.J Clin Neurosci 2009;16:867-76.

22. Takayanagui OM. Albendazole therapy for neurocysticercosis. Neurology 1998:50:834-5.

23. Verma A, Prasad KN, Cheekatla SS, Nyati KK, PaliwalVK, Gupta RK. Immune response in symptomatic and asymptomatic neurocysticercosis. Med Microbiol Immunol 20I I;200:255-6I.

24. Wallin MT, Kurtzke JF. Neurocysticercosis in the United States: Review of an important emerging infection. Neurology 2004;63:1559-64. 